\title{
Intravenous Contrast Media in Uroradiology: Evaluation of Safety and Tolerability in Almost 50,000 Patients
}

\author{
Gunnar Wendt-Nordahl ${ }^{\mathrm{a}}$ Harald Rotert ${ }^{\mathrm{b}}$ Lutz Trojan ${ }^{\mathrm{a}}$ \\ Maurice Stephan Michel ${ }^{\mathrm{a}}$ Candida R. Peters ${ }^{c}$ Peter Alken ${ }^{\mathrm{a}}$ Thomas Knoll ${ }^{\mathrm{a}}$ \\ ${ }^{a}$ Department of Urology, University Hospital Mannheim, Mannheim, ${ }^{b}$ Department of Radiology, \\ Krankenhaus Nordwest, Frankfurt, and ' ${ }^{\mathrm{G}}$ uerbet GmbH, Sulzbach, Germany
}

\section{Key Words}

Contrast media, safety $\cdot$ lobitridol .

Intravenous urography, adverse events

\begin{abstract}
Ojective: To evaluate the frequency of adverse events and possible risk factors after the administration of an intravenous contrast medium (CM), iobitridol, in a large multicentre postmarketing surveillance study. Subjects and Methods: A total of 49,975 patients undergoing intravenous urography were included in this study. A water-soluble, non-ionic CM (iobitridol, $1 \mathrm{ml} / \mathrm{kg}$ body weight) was administered. Age, sex, indication for the actual examination and adverse events were documented. Also, high-risk patients were identified. Results: Of the 49,975 patients, 28,336 (56.7\%) were males and 21,639 (43.3\%) females. Indications for urography were mainly urolithiasis (39.1\%), inflammatory diseases (25.6\%) and tumours (13.9\%). Additionally, 7.4\% were risk patients: $1.9 \%$ with creatinine $>1.5 \mathrm{mg} / \mathrm{dl}(0.1 \%$ were on haemodialysis); $0.8 \%$ had a history of previous allergic reactions to $C M$, $3.7 \%$ pre-existing asthma or allergies and $2 \%$ other risk factors against CM examination. Only $0.9 \%$ of the patients experienced acute adverse events that were non-serious and transient. Less than $0.1 \%$ of the patients experienced vomit-
\end{abstract}

ing, dizziness or cardiovascular problems. Only 1 patient developed an anaphylactic shock but recovered fully under treatment. $A \chi^{2}$ analysis revealed that adverse reactions occurred in patients with pre-existing renal insufficiency or allergies. Significantly more females had contrast-agent-related symptoms compared to men. Patients undergoing urography for urolithiasis had significantly fewer symptoms compared to patients with other indications. Conclusion: Iobitridol is clinically safe and well tolerated in urography as demonstrated in this study of a large patient population, producing mainly minor symptoms as adverse events. However, caution is advised when administering iobitridol to high-risk patients.

Copyright $\odot 2006$ S. Karger AG, Basel

\section{Introduction}

Intravenous water-soluble contrast media $(\mathrm{CM})$ are widely used for routine upper urinary tract investigation. Although toxicity and tolerability of CM have been improved, adverse events such as nausea or dizziness occur regularly. Pre-existing renal insufficiency can also deteriorate further, after the administration of CM. Low-osmolar contrast agents in animals indicate that these

\section{KARGER}

Fax +4161306 1234

E-Mail karger@karger.ch

www.karger.com
(C) 2006 S. Karger AG, Basel

$1011-7571 / 06 / 0155-0358 \$ 23.50 / 0$

Accessible online at:

www.karger.com/mpp
Thomas Knoll, MD, PhD

Department of Urology, University Hospital Mannheim, Theodor-Kutzer-Ufer 1-3 DE-68135 Mannheim (Germany)

Tel. +49621383 4080, Fax +49621383734080

E-Mail t.knoll@uro.ma.uni-heidelberg.de 


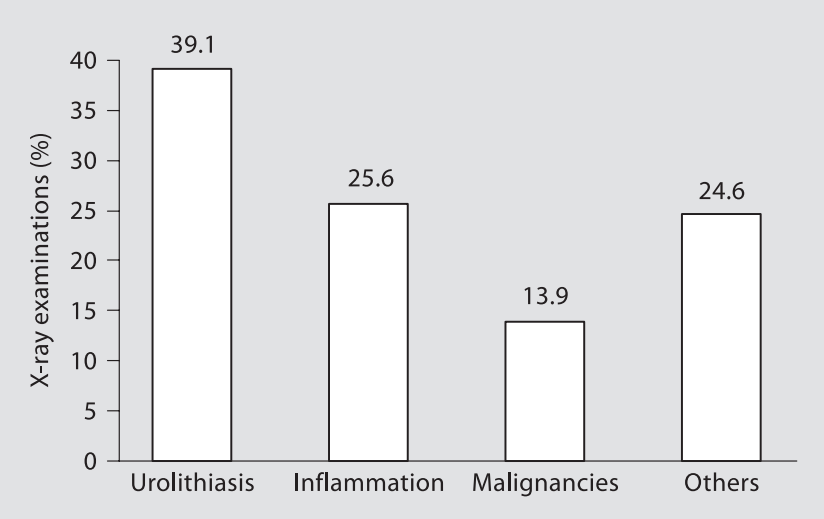

Fig. 1. Indications for intravenous urography (percentage of total number of examinations).

agents may affect kidney function less severely than highosmolar CM [1]. Low-osmolar CM are also better tolerated by the patient than high-osmolar substances, especially with regard to non-serious adverse events [2-4]. Iobitridol is a tri-iodinated low-osmolar non-ionic CM that exhibits excellent tolerability and low toxicity in intravenous urography and has been approved in Germany since $1996[5,6]$. In this large multicentre postmarketing surveillance study with iobitridol we aim to quantify the incidence of adverse events and identify possible risk factors.

\section{Subjects and Methods}

A total of 49,975 patients mainly undergoing intravenous urography in a routine clinical setting were included into this German multicentre postmarketing surveillance study. The lowosmolar, non-ionic CM iobitridol (Xenetix ${ }^{\circledR}$ from Guerbet GmbH, Sulzbach, Germany) was administered as a bolus injection $(1 \mathrm{ml} /$ $\mathrm{kg}$ body weight).

Age, sex and indication for the examination as well as the type of examination were documented. High-risk patients were identified; it included those with serum creatinine $>1.5 \mathrm{mg} / \mathrm{dl}$, previous reactions to $\mathrm{CM}$, prevalence of other allergies or lung diseases. After administration of iobitridol, adverse events were documented with respect to symptoms, duration and type of treatment needed as well as potential causality due to CM. Delayed reactions up to $24 \mathrm{~h}$ were also documented. Adverse event coding was done according to the World Health Organization Adverse Reaction Terminology. Classification into serious or non-serious adverse events was done according to guidelines: International Conference on Harmonization, World Health Organization, Food and Drug Administration. Statistical analysis was performed with the SPSS software package, using ANOVA and $\chi^{2}$ test. A p value $<0.05$ was considered to be of statistical significance.

Adverse Reactions following Intravenous Contrast Media

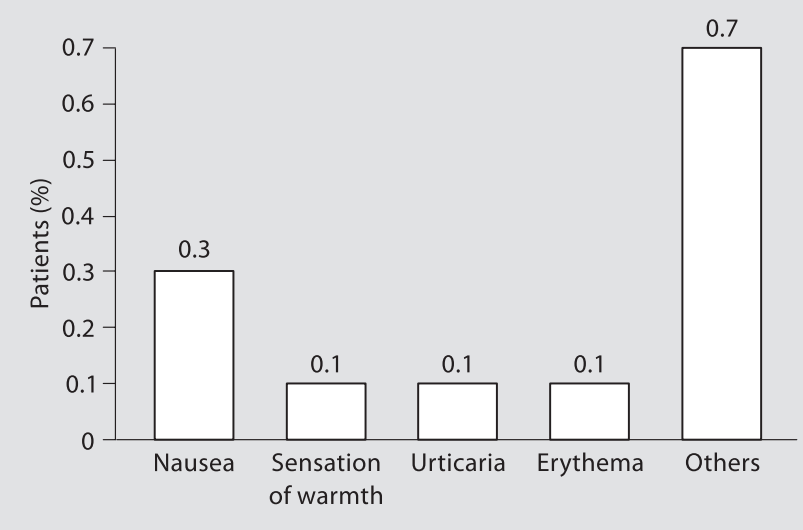

Fig. 2. Rate of adverse events within $24 \mathrm{~h}$ following iobitridol administration.

\section{Results}

Of the 49,975 patients, 28,336 (56.7\%) were males and $21,639(43.3 \%)$ females; the mean age was 56.6 years (range 3-101 years). Intravenous urography was performed in $98 \%$ of the patients, and the remaining $2 \%$ had either retrograde or other urological examinations. Indications for intravenous urography were mainly urolithiasis $(39.1 \%)$, inflammatory diseases $(25.6 \%)$ and tumours (13.9\%; fig. 1). Other frequent indications were prostatic diseases $(6.0 \%)$ or haematuria (3.1\%). Among those defined as high-risk patients (7.4\%), there were $1.9 \%$ with a creatinine level $>1.5 \mathrm{mg} / \mathrm{dl}$ ( $0.1 \%$ were on haemodialysis), $0.8 \%$ had a history of previous allergic reactions to contrast agents, $3.7 \%$ pre-existing asthma or allergies (more in females with $\mathrm{n}=1,035$ than in males with $\mathrm{n}=834$; $\mathrm{p}<$ 0.00001 ) and $2.0 \%$ other risk factors contra-indicating the administration of contrast agents.

The overall occurrence of acute symptoms after CM injection was $0.9 \%$. All symptoms were minor, rated as non-serious events with one exception. The incidence of most frequent non-serious events was nausea $(0.3 \%)$, sensation of warmth $(0.1 \%)$, urticaria $(0.1 \%)$ and dyspnoea ( $0.05 \%$; fig. 2). These reactions were transient in $81 \%$ of the cases; however, $51.1 \%$ of them required treatment. The symptoms were treated appropriately; medication was given in accordance with international guidelines. Less than $0.1 \%$ of the patients experienced vomiting, exanthema, dizziness, pruritus, flush or circulatory problems. The one serious event occurred in a 27 -year-old male who developed anaphylactic shock after intravenous urography. He received treatment including the ad- 


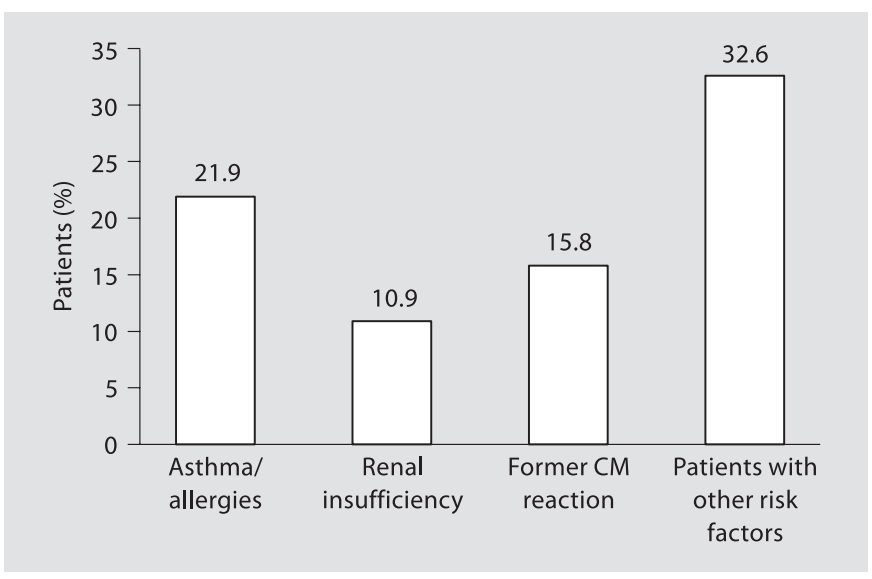

Fig. 3. Percentage of adverse events among high-risk patients.

ministration of corticosteroids and antihistaminics with a favourable outcome.

All adverse reactions happened within the first hour after $\mathrm{CM}$ administration, and delayed symptoms did not occur there after up to $24 \mathrm{~h}$. The $\chi^{2}$ analysis revealed that CM-related reactions occurred primarily in patients with renal insufficiency and pre-existing allergies/asthma (fig. 3). The number of patients that experienced adverse events in this group of patients was significantly higher than expected on the basis of overall incidence $(\mathrm{p}<$ $0.00001)$. Also, significantly more females $(1.2 \%)$ displayed CM-related adverse events compared to males $(0.6 \%)$. Patients undergoing urography for malignancies or benign prostatic hyperplasia had distinctly fewer symptoms than those with i.e. urolithiasis, haematuria, pain or urinary tract infections (fig. 4).

\section{Discussion}

Iobitridol is a water-soluble, tri-iodinated monomeric, non-ionic, low-osmolar contrast agent with a molecular weight of 835 and an octanol-water partition coefficient of 2.63. It is hydrophilic and a marker of extracellular fluid. It neither crosses the intact blood-brain barrier nor does it penetrate into cells. It possesses very low non-specific albumin-binding properties, and $>90 \%$ of the substance is eliminated by glomerular filtration without secretion or reabsorption. Iobitridol is rapidly excreted (93 $\pm 5 \%$ within the first $8 \mathrm{~h}$ ) in the urine $[7,8]$. Thus, with administration of iobitridol, contrast-agent-related ad-

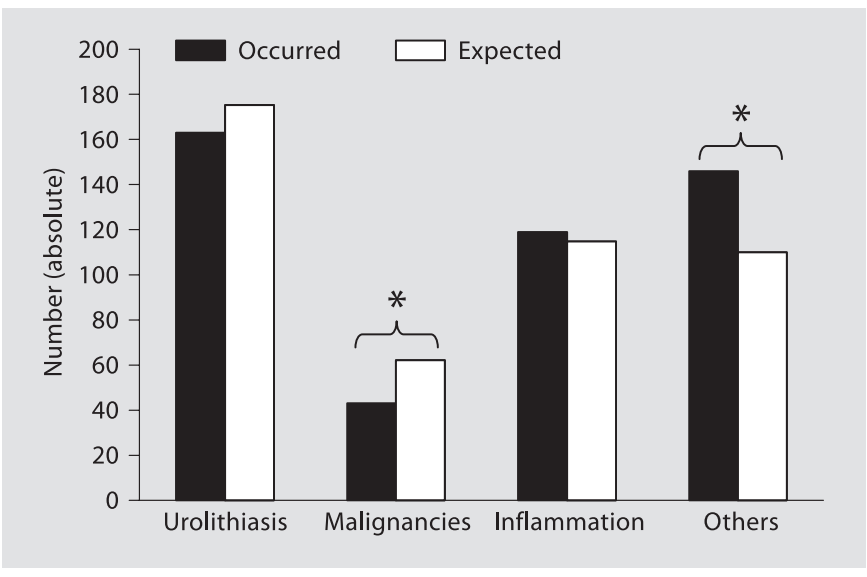

Fig. 4. Incidence of side-effects in relation to those expected in various indications. ${ }^{*} \mathrm{p}<0.001$.

verse events occur infrequently as seen in this study. Almost all symptoms were transient minor adverse events with no long-term effect on the patients' health. Patients with known allergies or impaired renal function bear a significantly higher risk of CM-related adverse events compared to those without these complications. Hence, precaution should be taken in patients with a history of allergic reactions following CM administration. The finding that the risk of adverse events is higher in patients with a pre-existing renal insufficiency can be attributed to slower elimination of the contrast agent.

The frequency of adverse reactions in our study is comparable with previously reported investigations in large radiological patient populations $[9,10]$. The observation that female patients in this study suffered significantly more adverse events than males is likely to be due to a higher prevalence of allergies in females. Since most published data on CM involved very low numbers of patients [2-5], a prediction on the clinical safety of CM in a large patient population can only be made with caution. The rate of serious adverse events in our larger multicentre study population was $0.002 \%$. This is in accordance with published results of $0.02-0.004 \%$ by Cochran et al. [11] and Katayama et al. [9].

Certain pre-existing diseases influence the rate of adverse events. Patients undergoing intravenous urography for suspected malignancies developed fewer adverse events than other patients. We do not have an explanation for this phenomenon, a reason might be that more 'acute' diseases like inflammation or urolithiasis have an influence on symptom production. It is therefore conceivable 
that accompanying illness negatively influences CM tolerability. The hypothesis that co-morbidity plays an important role must be further elucidated. The fact that patients in the 'other' group developed distinctly more reactions than calculated numbers could be accounted for by the heterogeneous compositions of this group.

Although the number of adverse events is lowered and the number of non-serious adverse events has decreased with the use of low-osmolar CM, serious adverse events still occur, though infrequently. The knowledge of the symptoms, the influence of pre-existing conditions and adequate treatment are crucial in this regard [11-13].

Since this study deals only with the occurrence of acute symptoms after the administration of iobitridol, no statement can be made about the long-term effect of the contrast agent, for example on the serum creatinine level. Further studies including the investigations of blood chemistry are therefore desirable.

\section{Conclusion}

Iobitridol is clinically safe and well tolerated in urography as demonstrated in this study of a large patient population, producing mainly minor symptoms as adverse events. CM administration was well tolerated by $99.1 \%$ of our patients. Hence, bearing risk patients in mind, this implies the clinical safety and diagnostic efficacy of iobitridol in urology.

\section{References}

$>1$ Deray G, Dubois M, Martinez F, Jacobs C: Radiocontrast nephrotoxicity in rats: a new model of acute renal failure. Am J Nephrol 1990;10:507-513.

-2 Deray G, Bellin MF, Zaim S, Raymond F, Grellet J, Jacobs C: Evaluation of the renal tolerance of Xenetix in patients with chronic renal failure. Nephron 1998;80:240.

$\checkmark 3$ Wasaki M, Kawamura H, Sugimoto J, Shimada M, Satoh Y, Tanaka E, Gemba M: Comparative toxic effects on iobitridol and iohexol on the kidney. Invest Radiol 1998;33: 393-400.

$\checkmark 4$ Wolf GL, Arenson RL, Cross A: A prospective trial of ionic vs nonionic contrast agents in routine clinical practice: comparison of side effects. Am J Roentgenol 1989;152:939944.
$>5$ Fournier PJ, Steinbrich W, Freitag P, Voegeli: Evaluation of the clinical safety and efficacy of iobitridol (Xenetix) in intravenous urography. Eur Radiol 1996;23:185-189.

6 Meiss L, Bakker AJ, Falke THM, van Waes PFGM: Clinical safety and efficacy of iobitridol in urography. Acta Radiol Suppl 1996;400:68-71.

7 Dencausse A, Violas X, Feldman H, Havard P, Chambon C: Pharmacokinetic profile of iobitridol. Acta Radiol Suppl 1996;400:2534.

$>8$ Idee JM, Balut C, Beaufils H, et al: Pharmacologic profile of iobitridol, a nonionic iodinated contrast medium. Acta Radiol Suppl 1996;400:35.

$\checkmark 9$ Katayama H, Yamaguchi K, Kozuka T, Takashima T, Matsuura K: Adverse reactions to ionic and nonionic contrast media. Radiology 1990;175:621-628.
10 Petersein J, Peters CR, Wolf M, Hamm B: Results of the safety and efficacy of iobitridol in more than 61,000 patients. Eur Radiol 2003; 13:2006-2011.

11 Cochran ST, Bomyea K, Sayre JW: Trends in adverse events after intravenous administration of contrast media. Am J Radiol 2001; 176:1385-1388.

12 Lasser EC, Lyon SG, Berry CC: Reports on contrast media reactions: analysis of data from reports to the US Food and Drug Administration. Radiology 1997;203:605-610.

13 Spring DB, Bettmann MA, Barkan HE: Deaths related to iodinated contrast media reported spontaneously to the US Food and Drug Administration, 1978-1994: effect of the availability of low-osmolality contrast media. Radiology 1997;204:333-337. 